\title{
Índices de Medida de Flexibilidade
}

Heitor Mansur Caulliraux

Professor do Programa de Engenharia de Produção, COPPE/UFRJ.

Programa de Engenharia de Produção COPPE/UFRJ - C.P. 68.507 - CEP 21.945

Rio de Janeiro - RJ

Maria das Graças Bruno Marietto

Angel G.Coca-Balta

Doutorandos do Programa de Engenharia de Sistem ase Computação, COPPE, UFRJ.

Programa de Engenharia de Produção COPPE/UFRJ - C.P. 68.507 - CEP 21.945

Rio de Janeiro - RJ

Palavras-chave: Desempenho industrial, avaliação do sistema produtivo, modernização industrial

Key words: Industrial performance, production systems assessments, industrial modernization

RESUMO:

ÍNDICES DE MEDIDA DE FLEXIBILIDADE: Este trabalho apresenta uma resenha sobre índices para medição de flexibilidade de um sistema de produçāo. Existem inúmeros fatores que influenciam a flexibilidade em uma indústria. Eles são inerentes ao tipo de sistema de produçāo instalado. Inicialmente, duas matrizes são construídas: a primeira inter-relaciona índices com os fatores; a segunda matriz inter-relaciona fatores com as indústrias. Realiza-se um exame comparativo entre os elementos destas matrizes e o resultado deste exame é uma matriz onde as linhas representam os índices estudados e as colunas representam as indústrias. Desta forma será possivel analisar o desempenho destes indices nas indústrias pesquisadas.

\section{ABSTRACT:}

This is review of flexibility measurcment fechniques for production systcms. These measurement techniques depends on the particular production systems adopted by a plant.

We generate two matrices: $A$ and $B$. Matrix $A$ is about the interaction between indexes and factors; matrix $B$ is about the interaction between factors and plants. Then, we multiply matrices $A$ and $B$ to generate mat rix $C$, wihe let us cvaluate the indexes of each plant.

Rec. 04/91 Rev. 04/91 Apr. 06/91

PRODUÇĀO - Rio de Janeiro - Vol. 2 - No2 - março 1992 - p.p. 133, 143 


\section{Introdução}

A partir da década de 70, novos sistemas de produçãocomeça ram a ser desenvolvidos comodecorrência, principalmente, doavanço tecnológico. Este avanço permitiu que mudanças nos produtos manufaturados pudessem ser feitas em periodos de tempo relativamente pequenos. Os consumidores passaram a exigiruma ada ptação mais rápida por parte das indústrias, com relação à fabricação de novos produtos, que, por sua vez, passaram a ser produzidos em vários modelos, versões e tipos diferenciados.

As adaptações realizadas leva ram à criação de novos conceitos e técnicas para o planejamento da produção de sistemas de manufatura. Entre os mais importantes, podemos citar o Just in Time e o Sistema de Manufatura Flexível.

Um sistema de Manufatura Flexível (SMF) é definido como um sitema integrado voltado para a produção de bens discretos, formado basicamente por um conjunto de máquinas (geralmente máquinas de controle numérico), por um sistema automático de manipulação de materiais e por um sistema computacional de controle.

Os objetivos de um SMF são a alta produtividade e flexibilidade.

A forte concorrência estabelecida no mercado mundial justifica a importância da produtividade. Já a flexibilidade é indispensável para a adaptação da indústria em relaçãoà exigência dos consumidores. Quando da avaliação de um SMF, é necessário considerara produtividade e a flexibilidade. Entretanto, neste trabalho nos concentraremos na análise da flexibilidade.

\section{Flexibilidade-sumário de tipos de flexibilidade encontrados na literatura}

Para que se possa falar em medida de flexibilidade precisamos, primeiramente, discorrer sobreos tipos de flexibilidade existentes. Um grande problema que aparece quando do estudo da flexibilidade é a sua definição, pois a mesma pode ser enfocada de várias formas.

Na literatura, a maior parte das referências é feita à classificação proposta por BROWNE et alli [1984]. Por este motivo, apresentamos a seguir um resumo desta classificaçāo.

\section{FLEXIBILIDADE DE MÁQUINA}

Definida como a habilidade de poder substituir ferramentas gastas, trocar ferramentas nomagazine e montarou remontar algum acessório de fixaçãosem interfenência ou longos tempos de set-up.

\section{FLEXIBILIDADE DE PROCESSO}

Definida como a habilidade de poder processar simultaneamente uma variedade de tipos de peças com pouca ou nenhuma inter-relação.

\section{FLEXIBILIDADE DE PRODUTO}

Definida como a habilidade de mudar para um novo conjuntode tipos de produtos a serem processados de forma rápida $e$ econômica. 


\section{FLEXIBILIDADE DE ROTEAMENTO}

Definida como a habilidade de mudar a seqüência das visitas às máquinas sem ter que modificar o conjunto de peças que está sendo processado.

\section{FLEXIBILIDADE DE VOLUME}

Definida como a habilidade de operar 0 sistema de maneira eficiente, mesmo diante de mudanças nos volumes de produção.

\section{FLEXIBILIDADE DE EXPANSĀO}

Definida como a capacidade do sistema de ser expandido de maneira fácil e modular.

\section{FLEXIBILIDADE DE OPERAÇĀO}

Definida como a capacidade de poder mudar a ordem das operaçōes no processamento de um tipo de peça.

\section{FLEXIBILIDADE DE PRODUÇĀO}

Definida comoa capacidade de produzir um amplo universo de tipos de peças.

\section{Medida de flexibilidade}

Um dos temas de ampla discussão quando da avaliação dos sistemas de produção é a medida de flexibilidade. Na literatura aparecem vários trabalhos desenvolvendoalgum tipode medida. Analisamos alguns destes índicese, a partir dos mesmos, desenvolvemos a metodologia proposta neste trabalho. A seguir apresentamos um resumo destes artigos.

\section{ABDEL-MALEK [1990]}

Neste artigo é proposta uma medida de flexibilidade que permite, quando da implantação de um SMF, escolher aquele que melhor se adapte às mudanças que por ventura venham a acontecer. Ou seja, é uma medida geral de flexibilidade para um SMF proposto.

Esta medida torna-se importante porque, em um planejamentode longo prazo, a análise da variação da demanda e do design do(s) produto(s) não pode ser feita quando da aquisição do SMF. Geralmente, a escolha do SMFéfeita baseada na experiência dos gerentes e no atual estado da arte da tecnologia.

Considerando como parâmetros básicos as rotas existentes para o desenvolvimento das operações num dado conjunto de peças ou produtos, os autores propóem um tipo de medida que permite relacionar, em termos de flexibilidade, dois conjuntos de tarefas. Este tipo de medida permite avaliar a flexibilidade do sistema com respeitoà mudança para um novo conjunto de tarefas.

Para obter esta medida geral, MALEK tenta relacionar indiretamente os oito tipos de flexibilidade definidos por BROWNE et alli [1984]. Como exemplo, quando são levadas em consideração as possibilidades viáveis para a fabricação de um produto, indiretamente está se considerando a flexibilidade de roteamento, mesmo sem determinar explicitamente um indice.

\section{MANDELBAUM E BRILL [1989]}

Estes autores apresentam medidas que têm a finalidade de quantificar a flexibilidade do sistema de manufatura. Basicamente, as medidas propostas estão relacionadas com um conjuntode tarefase por um conjun- 
to de máquinas, individual e coletivamente, e relacionando-as com um dado conjunto de tarefas.

São considerados os seguintes tipos de medida de flexibilidade:

1- Flexibilidade de uma máquina M relativamente a um conjunto de tarefas $T$. Este tipo de flexibilidade é medido usando a fórmula:

$$
\mathrm{F}(\mathrm{M})=\sum \mathrm{e}_{\mathrm{t} \in \mathrm{T}}\left(\ddot{M}, \mathrm{t}_{\mathrm{T}}\right){ }^{*} \mathrm{w}(\mathrm{t})
$$

Onde e(M,t) é a medida da eficácia da máquina $M$ para desenvolver a tarefa $t$, sendo que $0 \leq \mathrm{e}(\mathrm{M}, \mathrm{t}) \leq 1 ; \mathrm{w}(\mathrm{t})$ é o fator de importância da ta refa t no conjunto $\mathrm{T}$, talque $0 \leq w(t) \leq 1$ e $\sum_{1 \in T} w(t)=1$

No caso de considerar um sub-conjunto $\mathrm{T}^{\prime} \subset \mathrm{T}$, teremos

$$
F(M) t 1=\frac{\sum t \in T 1 e(M, t)^{*} w(t)}{\sum t \in T^{l} w(t)}
$$

2 - Flexibilidade de um grupo de máquinas relativamente a um conjunto de tarefas $T$.

- Medida otimista e medida pessimista da flexibilidade do grupo.

- Medida tipo - Hurwitz da flexibilidade do grupo

- Medida misturada da flexibilidade do grupo.

- Medida probabilística da flexibilidade do grupo.

- Medida redundante da flexibilidade do grupo.

É proposto um índice para medir flexibilidade de máquinas. Para tanto, é levada em consideração a importância de cada tarefa possível de ser realizada em uma máquina (ou grupo de máquinas) e pela eficiência com queesta máquina pode realizara referida tarefa. Este índice dará a flexibilidade de uma máquina em relação a um conjunto de tarefas a serem realizadas.

Entretanto, há casos em que é interessante calcular a flexibilidade de um grupo de máquinas, ao invés de uma máquina. Os autores propõem uma medida otimista e uma pessimista para a flexibilidade de um grupo de máquinas. Depois, consideram uma combinação linear convexa entre estas medidas, que é um valor intermediárioentre o otimismoe o pessimismo. Este valor intermediário é dado por $\alpha$, e é chamado de índice de otimismo.

Os a utores ta mbém aborda m o problema de medir a adaptabilidade, ou seja, "...quão bem uma máquina ou grupo de máquinas pode se adaptar a trocas".

\section{AZZONE E BERTELÈ [1989]}

Alguns autores fazem uma tentativa de estüdar a flexibilidade de um ponto de vista geral. Estes estudos tem por base avaliações econômico-estratégicas do valorda flexibilidade. Os índices propostos nestes casos são, no entanto, ainda muito precários.

No texto de Azzone e Bertelè (1989) é proposta uma forma de medir a flexibilidade baseada no mix de produção, nos tempos de máquina, no número de máquinas-ferramenta e na avaliação dos fluxos de caixa. $\mathrm{Na}$ primeira parte do modelo, é determinado o mix esperado de produtos com base no método Monte-Carlo, nas curvas de ciclo de vida do produtos e na distribuição esperada dos produtos em cada grupo. Após isto feito, ter-se-ia um quadro na evolução esperada da demanda. O segundo passo 
seria estimar as horas-máquinas necessárias para processar os volumes de produção (saídas da previsão dedemandajá realizada). Para isso, seriam tomados como base os tempos-máquina de operações semelhantes já ralizadas. A terceira fase do modelo, gera um quadro de máquinas necessárias para atender os itens acima. Finalmente, uma avaliação econômica (valor atual, taxa interna de retorno) érealizada afim dedefinir a lucratividade do investimento naquelas máquinas. $O$ valor resultante depende da flexibilidade dosistema escolhido. O modelo poderia ser usado para comparar alternativas (dada uma posiçāo estratégica) ou para estudar os efeitos na rentabilidade dos investimentos em função de mudanças estratégicas.

Outros autores têm tentado medir o valor estratégico da flexibilidade. Alguns trabalhos partem da premissa segundo a qual quanto maior a flexibilidade, maiores serão as possíveis decisões a serem tomadas no futuro. Estes autores se apóiam na Teoria das Decisões, (Fensterseifer, 1990; Hutchinson e Sinha, 1989). Estes autores, no entanto, não avançam significativamente na proposição de índices de medida concretos.

\section{TAYMAZ [1989]}

O autor constrói um modelo para um sistema formado por apenas uma máquina, objetivando interligar o conceito econômico de flexibilidade de volume com o conceito deflexibilidade de máquina. Comoosistema possui apenas uma máquina, flexibilidades como de roteamento, processo e de peças são desconsideradas, pois estão implícitas na flexibilidade de máquinas.

Em um sistema de produção, tem-se dois fatores cruciais, quais sejam: flexibilidade e produtividade. Taymaz representa a produtividade através do vetor $P=\left[p_{1}\right]_{n \prime}$ onde cada elemento $p_{j}$ representa uma "medida do esforço requerido para finalizar a operação $\mathrm{j}$ ".Éassumidoque esta medida será considerada em termos de unidade de tempo.

Com relação à flexibilidade, são estudadas as de máquina e de volume. Para calcular a flexibilidade de máquina são considerados dois parâmetros. O prìmeiro é o esforço decorrente da troca de jigs e/ou ferramentas. Este esforço, medido através de tempo,é representado pela matriz $F R=\left[a_{i j}\right] n n$. O elemento aij reflete o tempo para rearranjar a máquina quando na troca da operação i para a operação j.

A matriz $F S=\left[b_{k]}\right]$ mm determina o esforço da máquina (medido pelos custos diretos) para mudar para o item 1 da operação $k$. Existe uma relação direta entre os elementos de FR, FS e P. Ou seja, uma operação nas matrizes equivale a uma operação em $P$. Para analisar-se duas máquinas, $A$ e $B$, basta fazer a comparação abaixo:

\section{$\mathrm{FRa} \leq \mathrm{FRb}$ e FSa $5 \mathrm{FSb}$}

Se as desigualdades forem satisfeitas, entāo a máquina $A$ é mais flexível que a máquina $\mathrm{B}$. Com relação à produtividade, $\mathrm{O}$ mesmo raciocínio é válido. Caso a máquina A seja mais produtiva que a $\mathrm{B}$, então $\mathrm{Pa} \leq \mathrm{Pb}$.

Outro aspecto considerado é o "loading of machine tools", que é representado pelas matrizes OS $=\left[\mathrm{i}_{\mathrm{i} j}\right] \mathrm{nneST}=\left[\mathrm{S}_{\mathrm{k}]}\right] \mathrm{nm}$. Oelemento $0_{i j}$ determina o número de resets feitos na máquina quando da mudança da operaçāo i para a operaçãoj. Oelemento $S_{k 1}$ é dado pela forma abaixo:

$S_{k 1}=\left\{\begin{array}{l}1, \text { se houver reset na máquina para a } \\ \text { execuçāo do item } 1 \text { da operação } k \\ 0, \text { caso contrário }\end{array}\right.$

Atavés das matrizes FR, FS, OS, ST e do vetor $P$, TAYMAZ determina todas as características de produção, como por exemploo 
tempo total de produção para um batch dado, custo de matéria-prima, custo de estoque, tempo total de produção etc... Com base nestes dados da linha de produção é determinada a função custo TC tal que:

$$
\mathrm{TC}=\left[\operatorname{tr}\left(\mathrm{FR}^{\prime} \mathrm{OS}\right)+\mathrm{b}\left(\mathrm{P}^{\prime} \mathrm{OS}^{\prime} \mathrm{I}\right)\right](\mathrm{v} / \mathrm{b})\left(\mathrm{v}^{*} \mathrm{e}\right)+
$$
$b^{*} i[(V A / 2)+M C]+V^{*} M C+T F C+\operatorname{tr}\left(F S^{\prime} S T^{\prime}\right)$.

Onde tr é o trace operator que soma os elementos da diagonal de uma matriz quadrada, v é o volume de produção (em unidades), e e o fator de conversão do custo variável, VA'éovalor adicionado, i é o custo de armazenagem do estoque.

Através da derivada da função $\mathrm{TC}, \mathrm{ob}-$ tém-se a flexibilidade de volume. Esta, indica o quanto a função custo (TC) variará caso o volume de produção também varie.

\section{KUMAR [1987]}

Oautorapresenta um enfoqueaxiomático para desenvolver uma teoria da flexibilidade em sistemas de manufatura. Algumas medidas teóricas da informação são usadas para quantificar vários tipos de flexibilidade.

O primeiro critério para a avaliação é a dependência das opções disponiveis de escolha e do grau de liberdade com o qual pode-se fazer a escolha. Para tanto é considerada uma população estatística de $\mathbf{n}$ opçōes $(X 1, X 2, \ldots, X n)$ que são frações ou porções equivalentes às probabilidades de escolha.

Uma medida da flexibilidade proposta é dada pela fórmula:

$F 1(X 1, X 2, \ldots, X n)=\sum X i^{*} 1 n(X i)$,

onde $0^{*} 1 \mathrm{n} 0=0$ por definição. Esta medida permite avaliar a flexibilidade de um sistema segundo um critério operacional.

\section{Análise proposta}

Existem inúmeros fatores que influenciam a flexibilidade em uma indústria. Eles são inerentes ao ti po de sistema de produção instalado, Comoexemplo, em uma indústria de componentes eletrônicos o fator tempo de set-upé preponderante para que os tipos de flexibilidadesejam analisados. Nosartigos pesquisados, foram detectados os itens considerados para se mensurar a flexibilidade. Estes itens estão descritos a seguir:

1 - tempo de set-up

2 - importância que cada ta refa tem no sistema

3 - eficiência com que cada máquina pode realizar cada tarefa

4- rotas possiveis de manufatura dos produtos analisados

5 - custo de manuseią do estoque

6 - custo total de matérias primas

7 - total dos custos variáveis

8 - custo de execuçāo de itens de uma operação

9 - volume de produção.

A partir da determinação destes fatores, montam-se as matrizes de Oferta eDemanda.

\section{Matriz de Oferta}

$$
A=\left[a_{i j}\right]_{m s}
$$

onde $m$ é o número de tipos de flexibilidade e s o número de fatores analisados. Nesta matriz especifica-se o peso que cada fator tem na determinação dos índices de 
flexibilidade. Estes pesos são dados de acordo com as regras abaixo:

1: crucial para a flexibilidade

0 : irrelevante

Matriz de Demanda

$B=\left[b_{j k}\right]_{s r}$

tal que séo número de fatores analisados e ro número de indústrias.

Os elementos da matriz B foram obtidos através de uma pesquisa de campo. Nesta pesquisa foram levantados, juntoa um grupo de empresas, quais fatores influenciam diretamente a produção das mesmas. Esta relação foi traduzida a partir dos seguintes pesos:

\section{1: exerce influência na produção}

0: não exerce influência

Tem-se a seguinte matriz produto $C=$ $\left[C_{i k}\right]_{m r}$, onde seus elementos nos fornecem uma ordem de prioridade tanto na relação de um índice i com as $k$ indústrias, como na relação de uma indústria $\mathrm{k}$ com os índices $\mathrm{i}$. Considere a tabela abaixo para efetuar a multiplicação $C=A \times B$ :

\begin{tabular}{|c|c|c|}
\hline $\mathrm{a}_{\mathrm{ij}}$ & 0 & 1 \\
\hline 0 & 0 & 0 \\
\hline 1 & $1 / \mathrm{n}$ & 1 \\
\hline
\end{tabular}

tal que $\mathrm{n}$ é a quantidade de fatores. Quando algum fator $\mathrm{j}$ for considerado pelo índice i mas não influenciar no sistema de produção da indústria $k$, refletiremos esta relação através do valor $1 / \mathrm{n}$. Isto objetiva diferenciar da situação na qual este fator é importante para a indústria, mas não é considerado pelo índice k. Entretanto, a influência decorrente desta relação não dispensa uma análise mais especifica quando do estudo final.

Com o intuito de testar a metodologia aqui proposta, geraremos as matrizes A e B.

A montagem da matriz A é feita através do relacionamento existente entre os 5 índices de medida de flexibilidade estudados e os 9 fatores considerados. Após analisar estes índices, caso algum fator influenciasse na sua formaçāo, este teria peso 1 . Caso contrário, peso 0 . Temos portanto a seguinte matriz A:

$\begin{array}{lrrrrrrrrr} & \text { F1 } & \text { F2 } & \text { F3 } & \text { F4 } & \text { F5 } & \text { F6 } & \text { F7 } & \text { F8 } & \text { F9 } \\ \text { Malek } & 1 & 0 & 0 & 1 & 0 & 0 & 0 & 0 & 0 \\ \text { Mandelbaun e Brill } & 0 & 1 & 1 & 0 & 0 & 0 & 0 & 0 & 0 \\ \text { Azone e Bertelè } & 0 & 0 & 1 & 1 & 0 & 0 & 0 & 0 & 1 \\ \text { Taymaz } & 1 & 0 & 0 & 0 & 1 & 1 & 1 & 1 & 1 \\ \text { Kumar } & 0 & 1 & 0 & 0 & 0 & 0 & 0 & 0 & 0 .\end{array}$

A partir dos dados coletados durante a pesquisa decampo"Matriz Tecnológica para a Indústria de Processamento de Dados" (1) será gerada a matriz B. Nesta pesquisa foram levantados dados em 15 empresas da indústria de informática (computadores e periféricos), tendo comoobjetivo traçar uma "radiografia" tecnológica do setor. A pesquisa cobriu as fases de:

a) definição dos pmodutos (estratégia de negócio, análises de mercado, etc);

b) projeto de hardware e software;

(1) Este projeto foi conduzido pelo IEI/UFRJ, COPPE/UFRJ e IE/UNICAMP, com financiamento da Secretaria de Ciência e Tecnologia e Desenvolvimento Econôm ico do Estado de São Pauloe do CNPq. 
c) produção;

d) comercialização e assistência técnica;

e) estrutura de custo, relaçõescom os fornecedores e estrutura ocupacional.

Para este trabalho, usaremos dois conjuntos de dados. Em primeirolugar, os dados relativos à posição mercadológica / competitiva de cada produto. Estes dados, desde queconvenientemente analisados, permitem levantar as principais dimensões competitivas enfatizadas por cada empresa para o binômio produto/mercado.

Do conjunto das possiveis dimensōes competitivas, um subconjunto diz respeito às ca racterísticas dos sitemas de produçãoe, uma parte deste último subconjunto, se relaciona com as questōes de flexibilidade (ver figura a seguir).

Neste diagrama estão representadas as empresas A, B e N que competem no mesmo mercado através de produtos substituíveis (em algum grau) entre si. A dimensão competitiva 1 (D.C.1) poderia ser, por exemplo, - ocusto / preço do produto. Neste diagrama, por simplificação, a D.C. 1 é função do sistema de produção - capacidade de minimização dos custos.

Algumas D.C.'s são função de mais de um dos sistemas da empresa. Por exemplo, a capacidade de variar, com eficiência, o volume de produtos comercializados é função (ao menos) da produção e de vendas e marketing (canais de distribuição).

Neste trabalho, estuda remos o conjunto das variáveis dos sistemas de produção capaz de viabilizar uma competição baseada na flexibilidade deste sistema.

O segundo conjunto de dados usados, trata dos sistemas de produção deste setor industrial. Para se chegar a um entendimento doque é (e poderia ser) a flexibilidade na informática, é necessário que se entenda a lógica destes sistemas de produção. É necessáriotambém, dispor de índices quantitativos dos sistemas existentes (tamanho e composição dos mix's de produção, tempos médios de set-up, lead-time's na produção, entre outros).

Por questões de espaço, descreveremos dois casos típicos encontrados neste setor industrial. A partir das descriçōes, criaremos

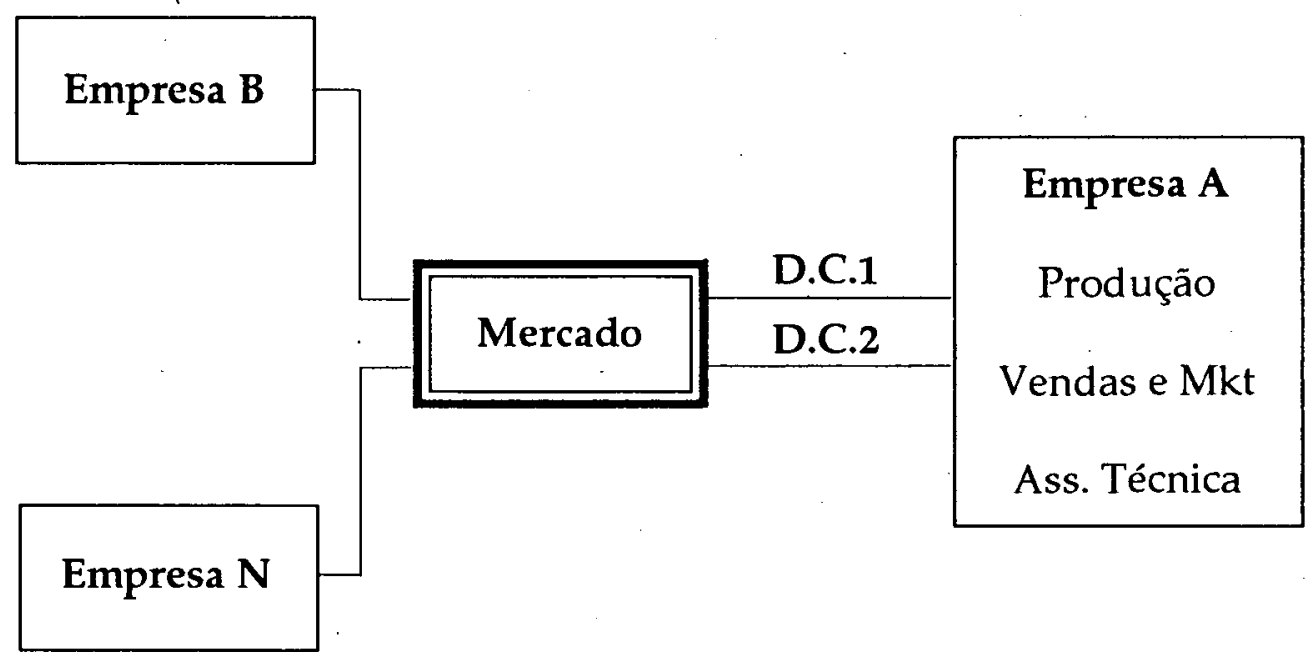


o vetor $B_{1}$ (coluna da matriz $B$ ) para cada situação para, em seguida, através da matriz $C$, identificarmos os métodos quantitativos mais adequados.

O primeiro caso é o da montagem do HDA (Hard Disk Assembly) de uma das multinacionais da informática instaladas no país. OHDA é um conjunto fechadocontendo discos magnéticos, cabeças de leitura e gravação, motores, base, etc. É.uma memória de massa usada como periférico de sistemas de computação de grande porte. A linha que estamos descrevendo é composta de uma série de postos de montagem semiautomáticos seguida de um conjunto de bancadas de teste. Esta linha entrega em torno de 2 centenas de produtos montadose testados por mês. O HDA é produzido sempre na mesma sequência e o sistema de transporte é mecanizado e interliga todas as bancadas. Este produto integra periféricos que são vendidos no país e no exterior. Em princípio, os volumes de produção são conhecidos com meses de antecedência $e$ são fixos no curto prazo.

Do ponto de vista da flexibilidade, a principal questão são os volumes de produção. Dois são os métodos possíveis para o aumento destes volumes: a) a umentar a taxa de utilização das bancadaseb) usarbancadas que duplicam a capacidade de produção dos gargalos. A partir desta descrição, chegamos à seguinte coluna da matriz B:

\section{set-up: 0}

importância relativa das tarefas no sistema: 0

eficiência comparada das máquinas: 0 rotas possiveis: 1

custo total de matérias prima: 1

custo de manuseio de estoques: 0

custos variáveis: 1

custos de execução de itens de uma operação: 1

volume de produção: 0
O segundo caso é o da produção de micro-computadores (vários modelos) e terminais de vídeo (vários modelos) em uma linha multiproduto. Esta empresa compete em mercados de elevada instabilidade tendo, como consequência, um mix de produção (composiçãoe volumes relativos) muito variado. O seu horizonte de planejamento é maior que 1 mês.

Como solução, a empresa adotou uma linha multi-produto do seguinte tipo (ver figura na página seguinte):

Cada pallet padrãoé composto das peças e partes que com porão determinado produto. O pallet é direcionado mecanicamente para a estação de trabalho que se encontrar vaga. Nesta estação o operário dispõe de todas as ferramentas necessárias para montar os produtos. Ao terminar a montagem, o operário recoloca o pallet na linha que leva o produto para as estações de teste.

A capacidade de produção (homens/ hora) é fixa nocurtíssi mo prazo e a eficiência do sistema éfunção dos set-up's nas estações e da habilidade dos operários. Esta situação nos leva ao seguinte vetor $B_{j}$ :

\section{set-up: 1}

importância relativa das tarefas no sistema: 0

eficiência comparada das máquinas: 1 rotas possiveis: 1

custo total de matérias prima: 1 custo de manuseio de estoques: 0 custos variáveis: 1

custo de execução de itens de uma operação: 0

volumes de produção: 1

Comoas matrizes A e Bjá estãomontadas, basta efetuarmosoexamecomparativoentre as mesmas, gerando a matriz C. Assim, temos: 


$\begin{array}{lll} & \text { Caso 1 } & \text { Caso 2 } \\ \text { Malek } & 1,111 & 2 \\ \text { Mandelbaun e Brill } & 0,222 & 1,111 \\ \text { Azone e Bertelè } & 1,222 & 3 \\ \text { Taymaz } & 4,222 & 5,111 \\ \text { Kumar } & 0,111 & 0,111\end{array}$

\section{Conclusão}

A aplicação dos índices selecionados na seção anterior nos fornece um retrato do sistema de produção estudado em relação à flexibilidade. Seria possivel, portanto, comparar diferentes sistemas de produção aplicados em situações de produto/mercado semelhantes.

No entanto, nos parece evidente que a melhor aplicação das diversas metodologias de medida de flexibilidade acontecerá na fase de projeto e seleçãodos equipamentos que irão compor um sistema de produção., Este tipodeaplicação deve se dar da seguinte forma:

$1^{\circ}$ - definição da relação produto/mercado e das dimensöes competitivas principais.

$2^{\circ}$ - a partir do item acima, definem-se as características genéricas do futuro sistema de produção.

$3^{\text {o }}$ - a partir do vetor coluna $\mathrm{Bj}$, seleciona-se $o$ indice mais adequado para este caso.

$4^{0}$ - aplicando-se este índice em cada um dos possiveis sistemas de prod ução (que por serem coerentes com o item 1, são passiveis de serem analisados pelo mesmo índice), obter-se-ão indicadores quantitativos que serão usados como auxílio na seleção final dos equipamentos e sistemas.

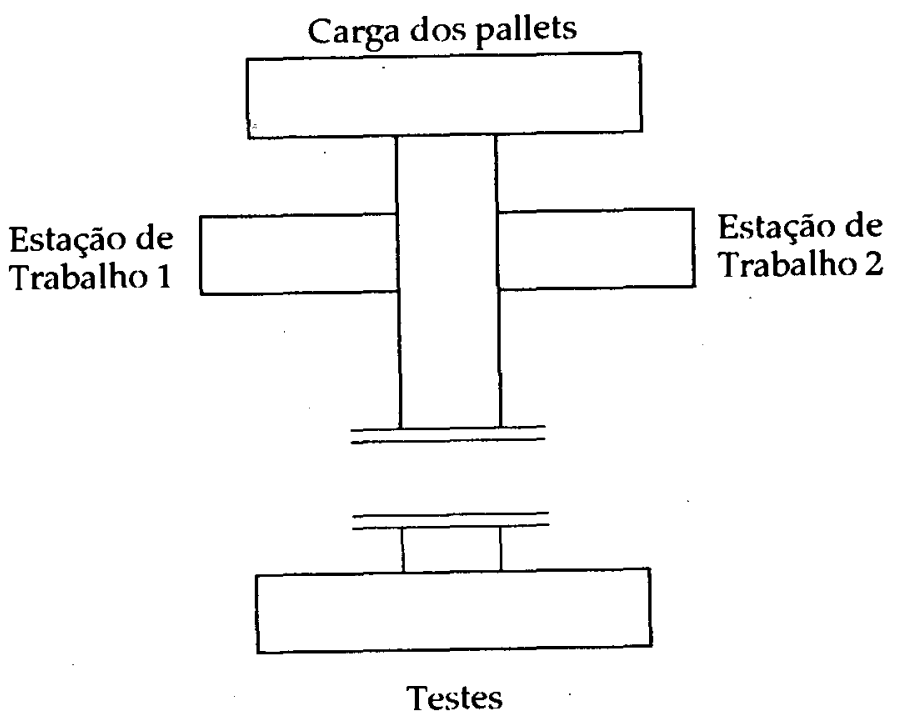




\section{Bibliografia}

BROWNE,J. et alli - - Classification of Flexible ManufacturingSystems, in The FMS Magazine, abril de 1984.

BULLINGER, H.J. e Warnecke, H.J. Toward the Factory of the Future, Proceedings of $8^{\circ}$ International Conference on Production Research and $5^{\circ}$ Working Conference of the FraunhoferInstitute of Industrial Engineering, editado por H. J. Bullingere H.J. Warnecke, 1985.

GRUPTA, Yash P. e Goyal, Sameer - Flexibility of Manufacturing Systems: Conceptsand Meadurements, EuropeanJournal of Operation Research, 43, 119-135, 1989.

HEGLAND, Donald E. - Manufacturing Systems for the Automated Factory, The Production Engineer, 42-47, agosto 1984.

KUSIAK, Andrew-Flexible Manufacturing Systemas: Methods and Studies, Studies in Management Science and Systems, vol. 12, editado por Andrew Kusiak, 1986.

AZZONE, Giovanni e Bertelè, Umberto Measuring the Economic Effectiveness of Flexible Automation: A New Approach, in Int. Journal of Production
Research, vol. 27, ṇo.5, páginas 735-746, 1989.

FENSTERSEIFER, Jaime E. - Um Modelo Conceitual para Avaliação de Investimento em Tecnologias Flexíveis de Produção, PPGA/UFRGS, Porto Alegre, 1990.

HUTCHINSON, George K. e Sinha, Diptendu - A Quantification of the Value of Flexibility", in "Journal of Manufacturing System, vol. 8, no.1, 1989.

HAYES, Robert H., Wheelwright, Steven C. e Clark, Kim B. -Dynamic Manufacturing - Creating the Learning Organization, Free Press, New York, 1988.

MANDELBAUN, P.H. e Brill, P.H. - Examples of Measurement of Flexibility and Adaptativity in Manufacturing Systems, in Journal of Operation Research Society, 1989, 40(6).

TAYMAZ, E. - Types of Flexibility in a Single Machine Production System, in Int. Journal of Production Research, 1989, 27 (11).

KUMAR, V. - Entropic Measures of Manufacturing Flexibility, in International Journal of Production Research, 25(7), 1987.

ABDEL-MALEK, L. e Wolf, C. - Evaluating Flexibility of Alternative FMS Design: A Comparative Measure, (preprint), IGLS, Austria, 1990. 